\title{
Stochastic Funding of a Defined Contribution Pension Plan with Proportional Administrative Costs and Taxation under Mean-Variance Optimization Approach
}

\author{
Charles I. Nkeki ${ }^{1, *}$ \\ ${ }^{1}$ Department of Mathematics, Faculty of Physical Sciences, University of Benin, Nigeria.
}

Received: 15 May 2014; Accepted: 22 September 2014

Editor: David G. Yu

\begin{abstract}
This paper aim at studying a mean-variance portfolio selection problem with stochastic salary, proportional administrative costs and taxation in the accumulation phase of a defined contribution (DC) pension scheme. The fund process is subjected to taxation while the contribution of the pension plan member (PPM) is tax exempt. It is assumed that the flow of contributions of a PPM are invested into a market that is characterized by a cash account and a stock. The optimal portfolio processes and expected wealth for the PPM are established. The efficient and parabolic frontiers of a PPM portfolios in mean-variance are obtained. It was found that capital market line can be attained when initial fund and the contribution rate are zero. It was also found that the optimal portfolio process involved an inter-temporal hedging term that will offset any shocks to the stochastic salary of the PPM.
\end{abstract}

Keywords mean-variance; stochastic salary; defined contribution; taxation; administrative costs; efficient frontier; parabolic frontier.

AMS 2010 subject classifications 91B28, 91B30, 91B70, 93E20

DOI: $10.19139 /$ soic.v2i4.82

\section{Introduction}

The problem of international Pay As You Go public pension scheme is making individuals public, corporate bodies and governments of many countries of the

\footnotetext{
${ }^{*}$ Correspondence to: Department of Mathematics, Faculty of Physical Sciences, University of Benin, P. M. B. 1154, Benin City, Edo State, Nigeria. Email: nkekicharles2003@yahoo.com
}

ISSN 2310-5070 (online) ISSN 2311-004X (print)

Copyright (C) 2014 International Academic Press 
world to encourage the development and advancement of defined contributory pension schemes. For example, in May 1981, Chile replaced her pension scheme known as Pay-As-You-Go retirement scheme with a private managed system through making compulsory contribution into their retirement account. Defined contribution pension schemes will play a prominent role in increasing the wealth of the nation and that of the plan members, since it is designed and tailored towards the members needs by ensuring that members make their input in the day-to-day management of the scheme. This paper used the modern portfolio theory-mean-variance optimization approach. Modern portfolio theory is a theory of financial analysis that focus on the maximization of portfolio expected return for a given amount of portfolio risk, in other words, minimize risk for a given level of expected portfolio return. There have been many studies on the maximization of expected utility of terminal wealth of PPMs in the accumulation phase of defined contribution pension schemes. See, for example, $[7,14,4,2,5,9,11,10,8,16$, $17,18]$.

In the context of DC pension plans, the problem of finding the optimal investment strategy with stochastic salary, proportional administrative costs and taxation on fund process under mean-variance efficient approach has not been reported in any published articles. [12, 21] assumed a constant flow of contributions into the pension scheme. In the literature, the problem of determining the minimum variance on trading strategy in continuous-time framework has been studied by [20, 1, 21, 19, 15] considered a mean-variance optimization problem in a discrete-time multi-period framework. They introduced the idea of stochastic control theory in solving mean-variance optimization problem. [22] determined a mean-variance in a continuous-time framework. They established the possibility of transforming the difficult problem of mean-variance optimization problem into a tractable one, by embedding the original problem into a stochastic linear-quadratic control problem, that can be solved using standard methods in statistics. These approaches have been extended and used by many in the financial literature, see for instance, [21, 3, 12, 6, 13]. In this paper, we study a mean-variance approach to portfolio selection problem with stochastic salary of a PPM in accumulation phase of a DC pension scheme using quadratic utility function. Nkeki (2012) considered a mean-variance portfolio selection problem with inflation hedging strategy for a defined contributory pension scheme. The efficient frontier was obtained for three asset classes which include cash account, stock and inflation-linked bond. In this paper, we assume that the salary of the PPM is a stochastic.

Closely related to this article is a paper by [18], who studied a mean-variance portfolio selection problem with stochastic salary and inflation protection strategy in the accumulation phase of a DC pension plan. The flow of contribution made by PPMs was assumed to be invested into a market that is characterized by a cash account, an inflation-linked bond and a stock. The inflation-linked bond was 
traded and used to hedge inflation risks associated with the investment. The paper aimed at maximizing the expected final wealth and minimizing its variance. They obtained efficient frontier for the three classes of assets that will enable PPMs to decide their own wealth and risk in their investment profile at retirement. In the paper, efficient frontier was found to be parabolic in shape, due to the present of initial capital and the existence of stochastic contributions of the PPM. But, [18] do not addressed wealth and portfolio process with proportional administrative costs and taxation, which we intend to address in this paper. In this paper, administrative cost are those cost incurred in the day-to-day running of the scheme.

The highlights of this paper are as follows:

- Portfolio with stochastic salary and proportional administrative costs for DC pension scheme was established.

- The fund process of a PPM is subjected to taxation while the contribution is tax exempt.

- The efficient frontier of the two classes of assets under a stochastic funding was obtained.

- We found the capital market line only when the initial fund and the contribution rate are zero.

The remainder of this paper is organized as follows. In section 2, we present the financial market models and the wealth process of the PPM involving administrative costs and taxation. Section 3 presents the dynamics of the wealth process of PPM with propositional administrative costs and taxation. In section 4, we presents the mean-variance approach. In section 5, we present the optimization of the portfolio process. Section 6 presents the expected wealth at the terminal period. In section 7, we presents the efficient frontier. Finally, section 8 concludes the paper.

\section{The Models}

Let $(\Omega, \mathcal{F}, \mathbf{P})$ be a probability space. Let $\mathbf{F}(\mathcal{F})=\left\{\mathcal{F}_{t}: t \in[0, T]\right\}$, where $\mathcal{F}_{t}=$ $\sigma(W(s): s \leq t)$, where the Brownian motion $W(t)=\left(W^{Y}(t), W^{S}(t)\right)^{\prime}, 0 \leq t \leq$ $T$ is a 2-dimensional process, defined on a given filtered probability space $(\Omega, \mathcal{F}, \mathbf{F}(\mathcal{F}), \mathbf{P})$, where $\mathbf{P}$ is the real world probability measure and $T$ the terminal time. $\sigma_{S}(t)$ and $\sigma_{Y}(t)$ are the volatility vectors of stock and PPMs salary process with respect to changes in source of the risk arising from the stock market, $W^{S}(t)$ and source of risk arising from inflation, $W^{Y}(t)$ respectively. Moreover, $\sigma_{S}(t)$ and $\sigma_{Y}(t)$ are the volatility vectors for the stock and salary process respectively, referred to as the coefficients of the market and are progressively measurable with respect to the filtration $\mathcal{F}$. 


\subsection{Financial models}

In this paper, we assume that the pension fund manager (PFM) faces a market that is composed by a risk-free asset (cash account) and risky asset, all of whom are trade-able. Therefore, the dynamics of the underlying assets are given in (1) and (2):

$$
\begin{gathered}
d S_{0}(t)=r(t) S_{0}(t) d t, S_{0}(0)=1, \\
d S(t)=S(t)\left(\mu(t) d t+\sigma_{S}(t) d W(t)\right), S(0)=s_{0}>0,
\end{gathered}
$$

where, $S(t)$ is stock price process at time $t, \mu(t)$ is the appreciation rate for stock, $\sigma_{S}(t)=\left(\sigma_{S_{1}}(t), \sigma_{S_{2}}(t)\right), r(t)$ is the nominal interest rate, $S_{0}(t)$ is the price process of the cash account at time $t$,

\subsection{The salary process of a PPM}

We assume in this paper that the salary process $Y(t)$ at time $t$ of the PPM is governed the by the dynamics

$$
d Y(t)=Y(t)\left(\beta(t) d t+\sigma_{Y}(t) d W(t)\right), Y(0)=y_{0}>0,
$$

where $\beta(t)>0$ is the expected growth rate of the salary of the PPM at time $t$ and $\sigma_{Y}(t)=\left(\sigma_{Y_{1}}(t), \sigma_{Y_{2}}(t)\right)$ is the volatility of the buying power of a PPM's salary. $\sigma_{Y_{1}}(t)$ is the volatility caused by the source of inflation, $W^{Y}(t)$ and $\sigma_{Y_{2}}(t)$ is the volatility caused by the source of uncertainty arising from the stock market, $W^{S}(t)$.

\section{The Wealth Dynamics}

Let $c>0$ be the proportion of the PPM salary that is contributed into the pension plan (which is deducted at source), then the amount of contributions made by the PPM is $c Y(t)$ at time $t$. Let $0 \leq \eta<1$ be the proportion of PPM's contribution the fund manager will receive as administrative costs. Then, the net contribution of the PPM at time $t$ is $\xi Y(t)$, where $\xi=c(1-\eta)$. Let $K(t)$ be the fund process of the PPM at time $t$ and $\Pi(t)$ be the portfolio process in stock at time $t$ and $\Pi_{0}(t)=K(t)-\Pi(t)$ is the fund invested in cash account at time $t$. In this paper, we assume for simplicity that $\beta(t), \sigma_{Y}(t), r(t), \mu(t)$ and $\sigma_{S}(t)$ are constant in time.

Therefore, the fund, $K(t)$ dynamic evolution under the investment policy $\Pi$ is

$$
\begin{aligned}
& d K(t)=(r K(t)+\Pi(t)(\mu-r)) d t+\Pi(t) \sigma_{S} d W(t), \\
& K(0)=K_{0} \in \mathcal{R}_{+},
\end{aligned}
$$




\subsection{The wealth dynamics with taxation and propositional administrative costs}

Since the contribution of the PPM is tax exempt as stated in Nigerian Pension Reform Act, 2004, we only subject the fund, $K(t)$ to tax at the rate, $\nu$. Therefore, the total wealth process defined as $X(t)=(1-\nu) K(t)+\xi Y(t)$ of the PPM is governs by the stochastic differential equation (SDE):

$$
\begin{aligned}
& d X(t)=(\lambda X(t)+\Pi(t)(\mu-r)+\xi Y(t)(\beta-\lambda)) d t+\left(\Pi(t) \sigma_{S}+\xi \sigma_{Y} Y(t)\right) d W(t), \\
& X(0)=x_{0}=(1-\nu) K_{0}+\xi y_{0} \in \mathcal{R}_{+},
\end{aligned}
$$

where $\lambda=\frac{r}{1-\nu}$. If $\nu=1$, it implies that entire fund wealth is paid to the government as tax, which is unrealistic. In that case, the total wealth depends only on the net contributions of the PPM. If $\nu=0$, it implies that the investor did not pay tax to the government. In order to avoid these trivial cases, we assume that $\nu \in(0,1)$. The case of $\nu=0$ is realistic in a society where taxation is free for investment portfolio. In this case, $\nu \in[0,1)$. The amount $x_{0}$ is the initial fund paid into PPMs account at the beginning of the planning horizon.

\section{The Mean-Variance Formulation}

In this section, we assume that the PPM invests his/her contributions through the PFM from time 0 to time $T$. The aim of the PPM is to maximize his/her expected terminal wealth and simultaneously minimize the variance of the terminal wealth. Hence, the PPM aim at minimizing the vector

$$
[-E(X(T)), \operatorname{Var}(X(T))]
$$

The mean-variance optimization problem is defined as

$$
\min _{\Pi} \Theta=[-E(X(T, \Pi)), \operatorname{Var}(X(T, \Pi))]
$$

See [18] for details.

Solving (6) is equivalent to solving the following equation

$$
\min _{\Pi} \Theta=[-E(X(T, \Pi(.)))+\psi \operatorname{Var}(X(T, \Pi(.)))], \psi>0,
$$

see [18].

$$
\min _{\Pi(.)} \Theta=E\left[\psi X^{2}(T)-\phi X(T)\right]
$$

where,

$$
\phi=1+2 \psi E(X(T)) .
$$

See [18] for details. (8) is known as a linear-quadratic control problem. Our aim is to solve the following (16):

$$
\min (\Theta(\Pi(.)), \psi, \phi)=E\left[\psi X(T, \Pi(.))^{2}-\phi X(T, \Pi(.))\right]
$$


subject to:

$$
\left\{\begin{array}{cc}
\Pi(.), & \text { set of admissible portfolio strategy } \\
X(.), \Pi(.), & \text { satisfy }(3) .
\end{array}\right.
$$

\section{The Optimization Problem}

In solving (10), we set $\omega=\frac{\phi}{2 \psi}$ and $V(t)=X(t)-\omega$, See [18] for details.

As already explained in [18], the problem is equivalent to solving

$$
\min _{\Pi(.)} \Theta(\Pi(.), \psi, \phi)=\left[\frac{\psi V(T)^{2}}{2}\right] .
$$

(11) is a standard optimal stochastic control problem. The process $V(t)$ follows the SDE:

$$
\left\{\begin{array}{l}
d V(t)=(\lambda(V(t)+\omega)+\Pi(t)(\mu-r)+\xi Y(t)(\beta-\lambda)) d t \\
+\left(\xi \sigma_{Y} Y(t)+\Pi(t) \sigma_{S}\right) d W(t) \\
V(0)=x_{0}-\omega
\end{array}\right.
$$

Let

$$
U(t, k, y)=\min _{\Pi(.)} E_{t, k, y}\left[\frac{\psi V(T)^{2}}{2}\right] .
$$

Then, the value function $U$ satisfies the following Hamilton-Jacobi-Bellman (HJB) equation:

$$
\left\{\begin{array}{l}
\min _{\Pi \in \mathbb{R}}\left\{U_{t}+\lambda(k+\omega) U_{k}+\Pi(t)(\mu-r) U_{k}-\xi y \lambda U_{k}+\xi y \beta U_{y}\right. \\
\left.+\frac{1}{2} \xi^{2} y^{2} \sigma_{Y} \sigma_{Y}^{\prime} U_{y y}+\xi y \sigma_{S} \sigma_{Y}^{\prime} \Pi(t) U_{k y}+\frac{1}{2} \sigma_{S} \sigma_{S}^{\prime} \Pi(t)^{2} U_{k k}\right\}=0 \\
U(T, k, y)=\frac{1}{2} \psi k^{2}
\end{array}\right.
$$

Assuming $U$ to be a convex function of $k$, then first order conditions lead to the optimal fraction of portfolio invested in risky asset at time $t$ :

$$
\Pi^{*}(t)=\frac{\left.-(\mu-r) U_{k}-\xi y \sigma_{S} \sigma_{Y}^{\prime} U_{k y}\right)}{\sigma_{S} \sigma_{S}^{\prime} U_{k k}} .
$$

Substituting (15) into (14), we have

$$
\begin{aligned}
& U_{t}+\lambda(k+\omega) U_{k}-\frac{(\mu-r)^{2} U_{k}^{2}}{2 \Sigma_{s s} U_{k k}}-\frac{\xi y(\mu-r) \Sigma_{s y} U_{y} U_{k y}}{\Sigma_{s s} U_{k k}}-\frac{\xi^{2} y^{2} \Sigma_{s y}^{2} U_{k y}^{2}}{2 \Sigma_{s s} U_{k k}} \\
& +\frac{1}{2} \xi^{2} y^{2} \sigma_{Y} \sigma_{Y}^{\prime} U_{y y}-\xi y \lambda U_{k}+\xi y \beta U_{y}=0,
\end{aligned}
$$

where, $\Sigma_{s s}=\sigma_{S} \sigma_{S}^{\prime}$ and $\Sigma_{s y}=\sigma_{S} \sigma_{Y}^{\prime}$.

In this paper, we assume a quadratic utility function of the form:

$$
U(t, k, y)=A(t) k^{2}+B(t) k y+C(t) y^{2}+D(t) k+E(t) y+F(t) .
$$


Finding the partial derivative of $U$ in (17) with respect to $t, k, k k, k y, y$, and $y y$, and then substitute into (16), we have the following system of ordinary differential equations (ODEs):

$$
\begin{gathered}
A^{\prime}(t)+2 \lambda A(t)-\frac{(\mu-r)^{2} A(t)}{\Sigma_{s s}}=0 \\
B^{\prime}(t)+\lambda B(t)-2 \xi \lambda A(t)+\xi \beta B(t)-\frac{(\mu-r)^{2} B(t)}{\Sigma_{s s}}-\frac{\xi(\mu-r) \Sigma_{s y} B(t)^{2}}{2 \Sigma_{s s} A(t)}=0 \\
C^{\prime}(t)-\xi \lambda B(t)+2 \xi \beta C(t)+\xi^{2} \sigma_{Y} \sigma_{Y}^{\prime} C(t)-\frac{(\mu-r)^{2} B(t)^{2}}{4 \Sigma_{s s} A(t)} \\
-\frac{\xi(\mu-r) \Sigma_{s y} B(t) C(t)}{\Sigma_{s s} A(t)}-\frac{\xi^{2} \Sigma_{s y}^{2} B(t)^{2}}{4 \Sigma_{s s} A(t)}=0 ; \\
D^{\prime}(t)+2 \lambda \omega A(t)+\lambda D(t)-\frac{(\mu-r)^{2} D(t)}{\Sigma_{s s}}=0 \\
E^{\prime}(t)+\lambda \omega B(t)-\xi \lambda D(t)+\xi \beta E(t)-\frac{(\mu-r)^{2} B(t) D(t)}{2 \Sigma_{s s} A(t)} \\
-\frac{\xi(\mu-r) \Sigma_{s y} B(t) E(t)}{2 \Sigma_{s s} A(t)}=0 ; \\
F^{\prime}(t)+\lambda \omega D(t)-\frac{(\mu-r)^{2} D(t)}{4 \Sigma_{s s} A(t)}=0
\end{gathered}
$$

with boundary conditions

$A(T)=\frac{1}{2} \psi, B(T)=C(T)=D(T)=E(T)=F(T)=0$.

Solving the systems of ODEs in (18), (19) and (21) using the boundary conditions, we have the following:

$$
\begin{gathered}
A(t)=\frac{1}{2} \psi e^{\left(2 \lambda-\theta^{\prime} \theta\right)(T-t)} \\
B(t)=\frac{2 \lambda \psi \xi e^{\left(2 \lambda-\theta^{\prime} \theta\right)(T-t)}\left(e^{\gamma t}-e^{\gamma T}\right)}{e^{\gamma T}(\lambda-\xi \beta+\gamma)+e^{\gamma t}(\xi \beta-\lambda+\gamma)}, \\
D(t)=\psi \omega\left(e^{\lambda T}-e^{\lambda t}\right) e^{\left(\lambda-\theta^{\prime} \theta\right)(T-t)-\lambda t},
\end{gathered}
$$

where $\theta=\left(0, \theta_{S}\right)^{\prime}, \theta_{S}=\frac{\mu-r}{\sqrt{\Sigma_{s s}}}$ and $\gamma=\sqrt{(\lambda-\xi \beta)^{2}-\frac{4 \xi^{2} \lambda(\mu-r) \Sigma_{s y}}{\Sigma_{s s}}}$.

We observe that our utility function $U$ is indeed convex, since

$$
U_{k k}=2 A(t)>0, U_{k y}=B(t)>0, U_{y y}=2 C(t)>0 .
$$

Now, substituting partial derivative of $U$ into (15), we have the following proposition:

\section{Proposition 1}

The investment strategy is given by

$$
\Pi^{*}(t)=-\frac{\mu-r}{\Sigma_{s s}}(k+\omega)+\frac{(\mu-r) \omega e^{-\lambda(T-t)}}{\Sigma_{s s}}-\left(\frac{(\mu-r) y}{2 \Sigma_{s s}}+\frac{\xi y \Sigma_{s y}}{2 \Sigma_{s s}}\right) f(t),
$$

where $f(t)=\frac{4 \xi \lambda\left(e^{\gamma t}-e^{\gamma T}\right)}{e^{\gamma T}(\lambda-\xi \beta+\gamma)+e^{\gamma t}(\xi \beta-\lambda+\gamma)}$. 
Hence, substituting $X^{*}(t)$ for $k+\omega$ and $Y(t)$ for $y$ in (28), we have the following:

$$
\Pi^{*}(t)=-\frac{(\mu-r)}{\Sigma_{s s}} X^{*}(t)+\frac{(\mu-r) \omega e^{-\lambda(T-t)}}{\Sigma_{s s}}-\left(\frac{(\mu-r) Y(t)}{2 \Sigma_{s s}}+\frac{\xi Y(t) \Sigma_{s y}}{2 \Sigma_{s s}}\right) f(t),
$$

At $t=0$, we have

$$
\Pi^{*}(0)=-\frac{(\mu-r)}{\Sigma_{s s}} x_{0}+\frac{(\mu-r) \omega e^{-\lambda T}}{\Sigma_{s s}}-\left(\frac{(\mu-r) y_{0}}{2 \Sigma_{s s}}+\frac{\xi y_{0} \Sigma_{s y}}{2 \Sigma_{s s}}\right) f(0) .
$$

\section{Expected Final Wealth for the PPM}

In this section we consider the expected wealth and the second moment of the expected wealth for the PPM at time $t$. The evolution of the optimal stochastic fund $X^{*}(t)$ for a PPM under optimal feedback control $\Pi^{*}(t)$ can be obtained by substituting (29) into (5) to obtain:

$$
\begin{aligned}
& d X^{*}(t)=\left(\left(\lambda-\theta^{\prime} \theta\right) X^{*}(t)+\theta^{\prime} \theta \omega e^{-\lambda(T-t)}+Z(t)\right) d t \\
& +\left(G(t)-\theta^{\prime} X^{*}(t)+\theta^{\prime} \omega e^{-\lambda(T-t)}\right) d W(t),
\end{aligned}
$$

where,

$$
\begin{gathered}
Z(t)=\left(\xi(\beta-\lambda)-\frac{\theta^{\prime} \theta}{2} f(t)-\frac{\xi(\mu-r) \Sigma_{s y}}{2 \Sigma_{s s}} f(t)\right) Y(t), \\
G(t)=\left(\xi \sigma_{Y}-\frac{\theta^{\prime}}{2} f(t)-\frac{\xi\left(\sigma_{S}^{\prime}\right)^{-1} \Sigma_{s y}}{2} f(t)\right) Y(t) .
\end{gathered}
$$

By applying Itô Lemma on (31), we obtain the SDE that governs the evolution of the square of optimal control $X^{*}(t)$ :

$$
\begin{aligned}
& d X^{* 2}(t)=\left(\left(2 \lambda-\theta^{\prime} \theta\right) X^{* 2}(t)+2 X^{*}(t)(Z(t)-G(t) \theta)\right. \\
& \left.+\left(G(t)+\theta^{\prime} \omega e^{-\lambda(T-t)}\right)\left(G(t)+\theta^{\prime} \omega e^{-\lambda(T-t)}\right)^{\prime}\right) d t \\
& +2 X^{*}(t)\left(G(t)-\theta^{\prime} X^{*}(t)+\theta^{\prime} \omega e^{-\lambda(T-t)}\right) d W(t) .
\end{aligned}
$$

Similarly, applying Itô Lemma on the product of $Y(t)$ and $X^{*}(t)$, we have

$$
\begin{aligned}
& d\left(Y(t) X^{*}(t)\right)=\left(\left(\beta+\lambda-\theta^{\prime} \theta-\sigma_{Y} \theta\right) Y(t) X^{*}(t)+\theta^{\prime} \theta \omega Y(t) e^{-\lambda(T-t)}\right. \\
& \left.+Z(t) Y(t)+\sigma_{Y} Y(t) G^{\prime}(t)+\sigma_{Y} \theta \omega Y(t) e^{-\lambda(T-t)}\right) d t \\
& +\left(\left(\sigma_{Y}+\theta^{\prime}\right) Y(t) X^{*}(t)+Y(t) G(t)+\theta^{\prime} \omega Y(t) e^{-\lambda(T-t)}\right) d W(t) .
\end{aligned}
$$

Taking the expectation on both sides of (31) and (32), we find that the expected value of the optimal wealth and the expected value of its square follow the following ODEs:

$$
\left\{\begin{array}{l}
d E\left(X^{*}(t)\right)=E\left(\left(\lambda-\theta^{\prime} \theta\right) X^{*}(t)+\theta^{\prime} \theta \omega e^{-\lambda(T-t)}+Z(t)\right) d t \\
E\left(X^{*}(0)\right)=x_{0}
\end{array}\right.
$$




$$
\begin{aligned}
& \left\{\begin{array}{l}
d E\left(Y(t) X^{*}(t)\right)=E\left(\left(\beta+\lambda-\theta^{\prime} \theta-\sigma_{Y} \theta\right) Y(t) X^{*}(t)+\theta^{\prime} \theta \omega Y(t) e^{-\lambda(T-t)}\right. \\
\left.+Z(t) Y(t)+\sigma_{Y} Y(t) G^{\prime}(t)+\sigma_{Y} \theta \omega Y(t) e^{-\lambda(T-t)}\right) d t \\
E\left(Y(t) X^{*}(0)\right)=x_{0} y_{0}
\end{array}\right. \\
& \left\{\begin{array}{l}
d E\left(X^{* 2}(t)\right)=E\left(\left(2 \lambda-\theta^{\prime} \theta\right) X^{* 2}(t)+2 X^{*}(t)(Z(t)-G(t) \theta)\right. \\
\left.+\left(G(t)+\theta^{\prime} \omega e^{-\lambda(T-t)}\right)\left(G(t)+\theta^{\prime} \omega e^{-\lambda(T-t)}\right)^{\prime}\right) d t \\
E\left(X^{* 2}(0)\right)=x_{0}^{2}
\end{array}\right.
\end{aligned}
$$

Solving the ODE (34), we find that the expected value of the wealth under optimal control at time $t$ is

$$
E\left(X^{*}(t)\right)=x_{0} e^{\left(\lambda-\theta^{\prime} \theta\right) t}+\omega e^{\left(\lambda-\theta^{\prime} \theta\right) t-\lambda T}\left(e^{\theta^{\prime} \theta t}-1\right)+\int_{0}^{t} E(Z(s)) e^{-\left(\lambda-\theta^{\prime} \theta\right)(s-t)} d s,
$$

Solving (35), we have

$$
\begin{aligned}
& \left.E\left(Y(t) X^{*}(t)\right)=x_{0} y_{0} e^{\alpha t}+y_{0} \omega\left(\theta^{\prime} \theta+\sigma_{Y} \theta\right) e^{\alpha t} \int_{0}^{t} e^{\beta s-\lambda(T-s)}\right) d s \\
& +e^{\alpha t} \int_{0}^{t} E(Z(s) Y(s)) d s+\sigma_{Y} e^{\alpha t} \int_{0}^{t} E\left(Y(s) G^{\prime}(s)\right) d s,
\end{aligned}
$$

where $E(Y(t))=y_{0} e^{\beta t}$ and $E\left(Y^{2}(t)\right)=y_{0}^{2} e^{\left(2 \beta+\sigma_{Y} \sigma_{Y}^{\prime}\right) t}, \quad \alpha=\beta+\lambda-\theta^{\prime} \theta-$ $\sigma_{Y} \theta$.

$$
E\left(X^{* 2}(t)\right)=x_{0}^{2} e^{\left(2 \lambda-\theta^{\prime} \theta\right) t}+Q_{1}(t) e^{\left(2 \lambda-\theta^{\prime} \theta\right) t}+\omega Q_{2}(t) e^{\left(2 \lambda-\theta^{\prime} \theta\right) t}+\omega^{2} Q_{3}(t) e^{\left(2 \lambda-\theta^{\prime} \theta\right) t},
$$

where,

$$
\begin{aligned}
& Q_{1}(t)=\xi\left(\beta-\lambda-\sigma_{Y} \theta\right)\left[2 x_{0} y_{0} \int_{0}^{t} e^{\left(\alpha-2 \lambda+\theta^{\prime} \theta\right) s} d s\right. \\
&+2 \int_{0}^{t} \int_{0}^{s} E(Z(\tau) Y(\tau)) e^{\left(\alpha-2 \lambda+\theta^{\prime} \theta\right) s} d \tau d s \\
&+2 \sigma_{Y} \int_{0}^{t} \int_{0}^{s} E\left(Y(\tau) G^{\prime}(\tau)\right) e^{\left(\alpha-2 \lambda+\theta^{\prime} \theta\right) s} d \tau d s \\
&\left.+2 \int_{0}^{t} E\left(G(s) G^{\prime}(s)\right) e^{-\left(2 \lambda-\theta^{\prime} \theta\right) s} d s\right], \\
& Q_{2}(t)=2 \xi y_{0}\left(\theta^{\prime} \theta+\sigma_{Y} \theta\right)\left(\beta-\lambda-\sigma_{Y} \theta\right) \int_{0}^{t} \int_{0}^{s} e^{\left(\alpha-2 \lambda+\theta^{\prime} \theta\right) s} e^{\beta \tau-\lambda(T-\tau)} d \tau d s \\
&+\quad 2 \int_{0}^{t} E(G(s)) \theta e^{-\left(\lambda-\theta^{\prime} \theta\right) s-\lambda T} d s,
\end{aligned}
$$

Stat., Optim. Inf. Comput. Vol. 2, December 2014. 
$Q_{3}(t)=e^{-2 \lambda T} \int_{0}^{t} \theta^{\prime} \theta e^{\theta^{\prime} \theta s} d s=e^{-2 \lambda T}\left(e^{\theta^{\prime} \theta t}-1\right)$.

At $t=T$, we have the expected terminal wealth of the PPM to be

$$
E\left(X^{*}(T)\right)=x_{0} e^{\left(\lambda-\theta^{\prime} \theta\right) T}+\omega\left(1-e^{-\theta^{\prime} \theta T}\right)+\int_{0}^{T} E(Z(s)) e^{-\left(\lambda-\theta^{\prime} \theta\right)(s-T)} d s,
$$

From (9) and (40) and the definition of $\omega$, we have that $\omega$ is a decreasing function of $\psi$ :

$$
\omega=\frac{e^{\theta^{\prime} \theta T}}{2 \psi}+x_{0} e^{\lambda T}+e^{\lambda T} \int_{0}^{T} E(Z(s)) e^{-\left(\lambda-\theta^{\prime} \theta\right) s} d s .
$$

It then follows that

$$
E\left(X^{*}(T)\right)=x_{0} e^{\lambda T}+e^{\lambda T} \int_{0}^{T} E(Z(s)) e^{-\left(\lambda-\theta^{\prime} \theta\right) s} d s+\frac{e^{\theta^{\prime} \theta T}-1}{2 \psi} .
$$

From (42), we observe that the expected terminal wealth of the PPM is the sum of the wealth that one would get investing the whole portfolio always in both the risky and risk-free asset plus the term, $e^{\lambda T} \int_{0}^{T} E(Z(s)) e^{-\left(\lambda-\theta^{\prime} \theta\right) s} d s$ that depends both on the goodness of the risky asset, riskless one, initial contribution, and expected growth rate of the contributions of the PPM, plus a term, $\frac{e^{\theta^{\prime} \theta T}-1}{2 \psi}$ that depends both on the goodness of the risky asset with respect to the risk-free one and on the weight given to the minimization of the variance. Thus, the higher the expected optimal terminal wealth value, for everything else being equal; the higher the variance minimization parameter, $\psi$, the lower its expected terminal wealth. In the same vain, the higher the growth rate of the contributions of PPM, the higher the terminal wealth of the PPM which is an intuitive result. We therefore conclude that the higher the Sharpe ratio $\theta^{\prime} \theta$ and the growth rate of salary of PPM, the higher the terminal wealth of the PPM.

If the PPM do not make contributions into the scheme (i.e., $\left.y_{0}=0\right)$, (42) becomes

\section{Proposition 2}

$$
E\left(X^{*}(T)\right)=x_{0} e^{\lambda T}+\frac{e^{\theta^{\prime} \theta T}-1}{2 \psi} .
$$

If the total wealth is tax free (i.e., $\nu=0$ ), then (42) becomes

$$
E\left(X^{*}(T)\right)=x_{0} e^{r T}+e^{r T} \int_{0}^{T} E(Z(s)) e^{-\left(r-\theta^{\prime} \theta\right) s} d s+\frac{e^{\theta^{\prime} \theta T}-1}{2 \psi} .
$$

The optimal portfolio can now be rewritten in-terms of $\psi$ as follows

$$
\begin{aligned}
& \Pi^{*}(t)=-\frac{(\mu-r) X^{*}(t)}{\Sigma_{s s}}+\frac{\mu-r}{\Sigma_{s s}} E\left(X^{*}(T)\right) e^{-\lambda(T-t)} \\
& +\frac{(\mu-r) e^{-\lambda(T-t)}}{2 \psi \Sigma_{s s}}+\left(\frac{\mu-r}{2 \Sigma_{s s}}+\frac{\xi \Sigma_{s y}}{2 \Sigma_{s s}}\right) Y(t) f(t) .
\end{aligned}
$$

This shows that the portfolio process can be express as a function of the terminal wealth of the PPM and $\psi$. Interestingly, if $\nu=1$, the portfolio process in the risky asset becomes

$$
\Pi^{*}(t)=-\frac{(\mu-r) X^{*}(t)}{\Sigma_{s s}}+\left.\left(\frac{\mu-r}{2 \Sigma_{s s}}+\frac{\xi \Sigma_{s y}}{2 \Sigma_{s s}}\right) Y(t) f(t)\right|_{\lambda=\infty}=\infty
$$




\section{Proposition 3}

If $\nu=1$ then the optimal investment strategy must be infinite.

This shows that the expected wealth and its associated risk are received by the tax collector, which is an impossible task. Obviously, the portfolio in the risky asset do not exists when $\nu=1$. If $\nu=0$ (i.e., when the portfolio is tax free), the portfolio process becomes

$$
\begin{aligned}
& \Pi^{*}(t)=-\frac{(\mu-r) X^{*}(t)}{\Sigma_{s s}}+\frac{\mu-r}{\Sigma_{s s}} E\left(X^{*}(T)\right) e^{-r(T-t)} \\
& +\frac{(\mu-r) e^{-r(T-t)}}{2 \psi \Sigma_{s s}}+\left(\frac{\mu-r}{2 \Sigma_{s s}}+\frac{\xi \Sigma_{s y}}{2 \Sigma_{s s}}\right) Y(t) f(t) .
\end{aligned}
$$

(45) is made up of two parts. The first part (which involve the first three terms) is the classical investment strategy for the PPM. The second part is the intertemporal hedging term that offset any shock to the stochastic salary of the PPM.

At $t=0$, we have

$$
\begin{aligned}
& \Pi^{*}(0)=-\frac{(\mu-r) x_{0}}{\Sigma_{s s}}+\frac{\mu-r}{\Sigma_{s s}} E\left(X^{*}(T)\right) e^{-\lambda T} \\
& +\frac{(\mu-r) e^{-\lambda T}}{2 \psi \Sigma_{s s}}+\left(\frac{\mu-r}{2 \Sigma_{s s}}+\frac{\xi \Sigma_{s y}}{2 \Sigma_{s s}}\right) y_{0} f(0) .
\end{aligned}
$$

Table 1: Initial investment for $\eta=0.05, \nu=0.02$

\begin{tabular}{llllll}
\hline$X^{*}$ & $T=1$ & $T=2$ & $T=5$ & $T=10$ & $T=20$ \\
\hline 0.0 & 0.1985 & 0.1927 & 0.1760 & 0.1503 & 0.1060 \\
0.1 & 0.2485 & 0.2417 & 0.2221 & 0.1919 & 0.1399 \\
0.2 & 0.2986 & 0.2908 & 0.2682 & 0.2336 & 0.1739 \\
0.3 & 0.3487 & 0.3398 & 0.3144 & 0.2752 & 0.2079 \\
0.4 & 0.3987 & 0.3889 & 0.3605 & 0.3169 & 0.2419 \\
0.5 & 0.4488 & 0.4379 & 0.4067 & 0.3586 & 0.2758 \\
\hline
\end{tabular}

Table 2: Initial investment for $\eta=0.05, \nu=0.07$

\begin{tabular}{llllll}
\hline$X^{*}$ & $T=1$ & $T=2$ & $T=5$ & $T=10$ & $T=20$ \\
\hline 0.0 & 0.1982 & 0.1921 & 0.1747 & 0.1480 & 0.1023 \\
0.1 & 0.2482 & 0.2411 & 0.2206 & 0.1892 & 0.1355 \\
0.2 & 0.2982 & 0.2900 & 0.2665 & 0.2304 & 0.1688 \\
0.3 & 0.3482 & 0.3390 & 0.3134 & 0.2716 & 0.2020 \\
0.4 & 0.3982 & 0.3879 & 0.3582 & 0.3128 & 0.2352 \\
0.5 & 0.4482 & 0.4369 & 0.4041 & 0.3540 & 0.2685 \\
\hline
\end{tabular}


Table 3: Initial investment for $\eta=0.05, \nu=0.10$

\begin{tabular}{llllll}
\hline$X^{*}$ & $T=1$ & $T=2$ & $T=5$ & $T=10$ & $T=20$ \\
\hline 0.0 & 0.1980 & 0.1918 & 0.1739 & 0.1465 & 0.0999 \\
0.1 & 0.2480 & 0.2407 & 0.2196 & 0.1874 & 0.1327 \\
0.2 & 0.2980 & 0.2895 & 0.2653 & 0.2283 & 0.1654 \\
0.3 & 0.3479 & 0.3384 & 0.3110 & 0.2693 & 0.1982 \\
0.4 & 0.3979 & 0.3873 & 0.3568 & 0.3102 & 0.2310 \\
0.5 & 0.4479 & 0.4362 & 0.4025 & 0.3511 & 0.2637 \\
\hline
\end{tabular}

Table 4: Initial investment for $\eta=0.05, \nu=0.20$

\begin{tabular}{llllll}
\hline$X^{*}$ & $T=1$ & $T=2$ & $T=5$ & $T=10$ & $T=20$ \\
\hline 0.0 & 0.1973 & 0.1904 & 0.1707 & 0.1409 & 0.0911 \\
0.1 & 0.2472 & 0.2390 & 0.2158 & 0.1807 & 0.1221 \\
0.2 & 0.2970 & 0.2876 & 0.2609 & 0.2205 & 0.1531 \\
0.3 & 0.3468 & 0.3362 & 0.3060 & 0.2603 & 0.1840 \\
0.4 & 0.3967 & 0.3848 & 0.3511 & 0.3001 & 0.2150 \\
0.5 & 0.4465 & 0.4334 & 0.3962 & 0.3399 & 0.2460 \\
\hline
\end{tabular}

Table 1-4 show the initial investment where the administrative costs is $5 \%$ and tax rate varies. Table 1 presents the initial investment for administrative costs, $5 \%$ and tax rate $2 \%$. Table 2 shows the value of the initial investment where the tax rate is $7 \%$. Table 3 and 4 show the initial investment value for tax rate $10 \%$ and $20 \%$, respectively. We observed that the initial investment strategy decreases as the tax rate increases. This shows that taxation has significant effect on the investment strategy.

\section{Efficient Frontier}

In this section, we derive the efficient frontier for the original mean-variance problem (6). Using (40), we have

$$
E\left(X^{*}(T)\right)=\tilde{x}_{0} \epsilon+\omega \epsilon a,
$$

where $\tilde{x}_{0}=x_{0}+\int_{0}^{T} E(Z(s)) e^{-\left(\lambda-\theta^{\prime} \theta\right)(s-T)} d s, \epsilon=e^{\left(\lambda-\theta^{\prime} \theta\right) T}, a=1-e^{-\theta^{\prime} \theta T}$. It implies that

$$
\omega=\frac{E\left(X^{*}(T)\right)-\tilde{x}_{0} \epsilon}{\epsilon a} .
$$

The second moment becomes of the PPM's terminal wealth is obtained as

$$
E\left(X^{* 2}(T)\right)=x_{0}^{2} \epsilon e^{\lambda T}+\epsilon Q_{1}(T) e^{\lambda T}+2 \epsilon \omega Q_{2}(T) e^{\lambda T}+\epsilon \omega^{2} Q_{3}(T) e^{\lambda T},
$$


Therefore, the variance can be expressed as a function of the expected optimal final return $E\left(X^{*}(T)\right)$ as:

$$
\begin{aligned}
\operatorname{Var}\left(X^{*}(T)\right) & =x_{0}^{2} \epsilon e^{\lambda T}+\epsilon Q_{1}(T) e^{\lambda T}+2 \epsilon\left(\frac{E\left(X^{*}(T)\right)-\tilde{x}_{0} \epsilon}{\epsilon a}\right) Q_{2}(T) e^{\lambda T} \\
& +\epsilon\left(\frac{E\left(X^{*}(T)\right)-\tilde{x}_{0} \epsilon}{\epsilon a}\right)^{2} Q_{3}(T) e^{\lambda T}-\left[E\left(X^{*}(T)\right)\right]^{2} \\
& =x_{0}^{2} \epsilon e^{\lambda T}+\epsilon Q_{1}(T) e^{\lambda T}+2 \epsilon\left(\frac{E\left(X^{*}(T)\right)-\tilde{x}_{0} \epsilon}{\epsilon a}\right) Q_{2}(T) e^{\lambda T}+\tilde{x}_{0}^{2} \epsilon^{2} \\
& +\left(\epsilon Q_{3}(T) e^{\lambda T}-\epsilon a\right)\left(\frac{E\left(X^{*}(T)\right)-\tilde{x}_{0} \epsilon}{\epsilon a}\right)^{2}+\frac{2 \tilde{x}_{0} \epsilon\left(\left(E\left(X^{*}(T)\right)-\tilde{x}_{0} \epsilon\right) \epsilon a\right.}{\epsilon a} \\
& =x_{0}^{2} \epsilon e^{\lambda T}+\tilde{x}_{0}^{2} \epsilon^{2}+\epsilon Q_{1}(T) e^{\lambda T} \\
& +\left(\epsilon Q_{3}(T) e^{\lambda T}-\epsilon a\right)\left[\frac{2\left(Q_{2}(T) e^{\lambda T}+\tilde{x}_{0} \epsilon a\right)}{\left(Q_{3}(T) e^{\lambda T}-a\right)}\left(\frac{E\left(X^{*}(T)\right)-\tilde{x}_{0} \epsilon}{\epsilon a}\right)\right. \\
& \left.+\left(\frac{\left(E\left(X^{*}(T)\right)-\tilde{x}_{0} \epsilon\right.}{\epsilon a}\right)^{2}\right] \\
& =L(T)+\left(\epsilon Q_{3}(T) e^{\lambda T}-\epsilon a\right)\left[\frac{Q_{2}(T) e^{\lambda T}+\tilde{x}_{0} \epsilon a}{\left(Q_{3}(T) e^{\lambda T}-a\right)}+\frac{\left(E\left(X^{*}(T)\right)-\tilde{x}_{0} \epsilon\right.}{\epsilon a}\right]^{2}
\end{aligned}
$$

where

$$
L(T)=x_{0}^{2} \epsilon e^{\lambda T}+\tilde{x}_{0}^{2} \epsilon^{2}+\epsilon Q_{1}(T) e^{\lambda T}-\frac{\left(Q_{2}(T) e^{\lambda T}+\tilde{x}_{0} \epsilon a\right)^{2}}{\left(Q_{3}(T) e^{\lambda T}-a\right)} .
$$

Therefore, the expected wealth and the standard deviation, $\sigma_{X^{*}(T)}$, at time $T$ are related by

$$
E\left(X^{*}(T)\right)=\tilde{x}_{0} \epsilon-\frac{\left(Q_{2}(T) e^{\lambda T}+\tilde{x}_{0} \epsilon a\right) \epsilon a}{Q_{3}(T) e^{\lambda T}-a}+\frac{\epsilon a \sqrt{\sigma_{X^{*}(T)}^{2}-L(T)}}{\sqrt{\epsilon Q_{3}(T) e^{\lambda T}-\epsilon a}} .
$$

Hence, the minimum possible variance, $\operatorname{Var}\left(X^{*}(T)\right)=L(T) \geq 0$, is achieved when the investor borrows money from the total amount of wealth at time $t=0$ for $T$ years, so that

$$
E\left(X^{*}(T)\right)=\tilde{x}_{0} \epsilon-\frac{\left(Q_{2}(T) e^{\lambda T}+\tilde{x}_{0} \epsilon a\right) \epsilon a}{Q_{3}(T) e^{\lambda T}-a} .
$$

We have that (52) will be a straight line when the initial wealth is zero i.e., $x_{0}=0$ which implies that initial contribution is zero as well (i.e., $y_{0}=0$ or $c=0$ or $\eta=1$ ). In that case the capital market line is

$$
E\left(X^{*}(T)\right)=\frac{\epsilon a \sigma_{X^{*}(T)}}{\sqrt{\epsilon Q_{3}(T) e^{\lambda T}-\epsilon a}} .
$$




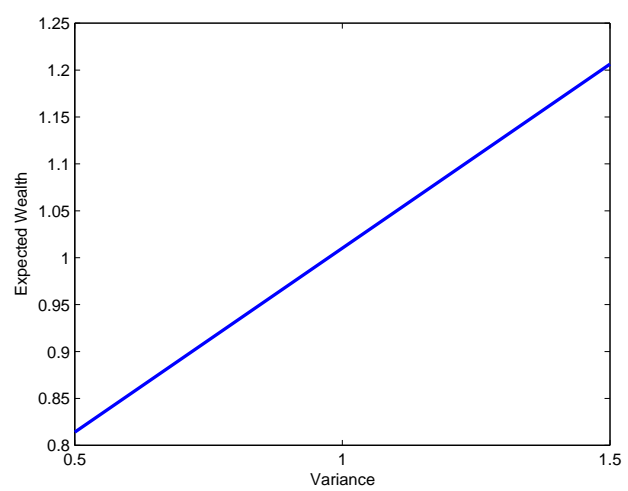

Figure 1. Efficient frontier. This is obtained by setting $\mu=0.09, r=0.02, \beta=0.0292$, $c=0.075, x_{0}=1, y_{0}=0.9, T=20, \sigma_{S}=(0.25,0.29), \sigma_{Y}=(0.18,0.20), \nu=0.05$, $\eta=0.01$.

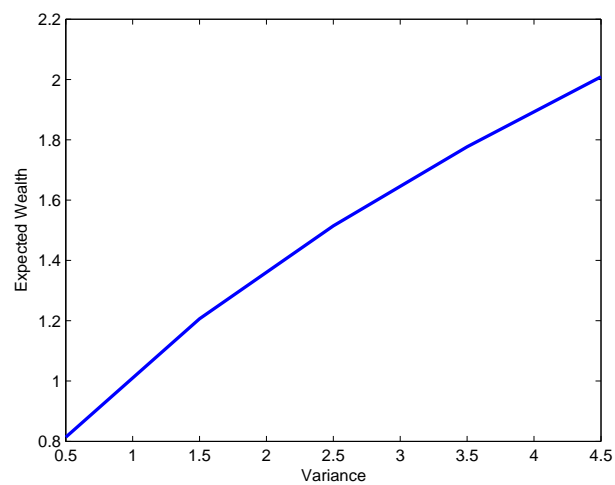

Figure 2. Efficient frontier. This is obtained by setting $\mu=0.09, r=0.02, \beta=0.0292$, $c=0.075, x_{0}=1, y_{0}=0.9, T=20, \sigma_{S}=(0.25,0.29), \sigma_{Y}=(0.18,0.20), \nu=0.05$, $\eta=0.01$.

The slope, $\frac{\epsilon a}{\sqrt{\epsilon Q_{3}(T) e^{\lambda T}-\epsilon a}}$, is the price of risk.

We observe that if $\lambda=\theta^{\prime} \theta$, the expected terminal wealth becomes

$$
\begin{aligned}
& \left.E\left(X^{*}(T)\right)\right|_{\lambda=\theta^{\prime} \theta}=\tilde{x}_{0}-\frac{\left.\left(e^{\lambda T}-1\right) Q_{2}(T)\right|_{\lambda=\theta^{\prime} \theta}+\tilde{x}_{0}\left(1-e^{-\lambda T}\right)^{2}}{e^{-\lambda T}\left(1-e^{2 \lambda T}\right)} \\
& +\frac{\left(1-e^{-\lambda T}\right) \sqrt{\sigma_{X^{*}(T)}^{2}-\left.L(T)\right|_{\lambda=\theta^{\prime} \theta}}}{\sqrt{e^{-\lambda T}\left(1-e^{2 \lambda T}\right)}} .
\end{aligned}
$$


Figure 1 and 2 show the efficient frontier of the portfolio choice. We observe in figure 1 that when the range of variance is $0-2$, we have a straight line graph. This shows that no fund (or very small amount of funds) was borrowed to finance the investment. In figure 2, we observe that the shape of the graph is parabolic in nature. This shows that the investor borrows money from the total amount of wealth at time $t=0$ for $T$ years. We observed from figure 1 and 2 the variance of the portfolio increase faster as the expected terminal wealth increases this must be due to the present of administrative costs and taxation, unlike the results obtained in [18] where administrative costs and taxation are not put into consideration.

\section{Conclusion}

This paper have studied stochastic finding of a defined contributory pension scheme with proportional administrative costs. The objectives are to determine total pension wealth that will accrued to the PPM and optimal investment strategies maximizing the expected terminal wealth and simultaneously minimizing the variance of the terminal wealth of a quadratic utility pension plan member. The financial market is made up of cash account and stock. The salary of the pension plan member is stochastic. The problem was formulated as a mean-variance optimization problem and was solved using dynamic programming approach.

The efficient frontier which was found to be nonlinear (i.e., possess a parabolic shape). The optimal investment strategies have two components. The first component depends ultimately on the risky asset and its correlation. The second component is proportional to the stochastic contributions of the pension plan member. It is the inter-temporal hedging terms that offset any shock to the stochastic finding overtime.

The optimal terminal wealth for the PPM was determined in this paper. The fund manager charge propositional administrative costs for the management of the fund. This costs is on the PPM stochastic contributions into the scheme. The fund process was not tax free.

A numerical illustrations of the efficient frontier was established in the paper.

Further research should be directed to include the following: time dependent administrative cost, time dependent taxation and stochastic riskfree rate of interest.

\section{REFERENCES}

1. I. Bajeux-Besnainou and R. Portait, Dynamic asset allocation in a mean-variance framework, Management Science, 44(1998), S79-S95.

2. P. Battocchio and F. Menoncin, Optimal pension management in a stochastic framework, Insurance: Mathematics and Economics, 34(2004), pp. 79-95. 
3. T. Bielecky, H. Jim, S. Pliska, and X. Zhou, Continuous-time mean-variance portfolio selection with bankruptcy prohibition, Mathematical Finance, 15(2005), pp. 213-244.

4. D. Blake, D. Wright and Y. Zhang, Optimal funding and investment strategies in defined contribution pension plans under Epstein-Zin utility, Discussion paper, The pensions Institute, Cass Business School, City University, UK (2008).

5. J. F. Boulier, S. J. Huang and G. Taillard, Optimal management under stochastic interest rates: The case of a protected defined contribution pension fund, Insurance: Mathematics and Economics, 28(2001), pp. 173-189.

6. M. Chiu and $\mathrm{D}$. Li, Asset and liability management under a continuous-time mean-variance optimization framework, Insurance: Mathematics and Economics, 39(2006), pp. 330-355.

7. G. Deelstra, M. Grasselli and P. Koehl, Optimal investment strategies in a CIR framework. Journal of Applied Probability, 37(2000), pp. 936-946.

8. P. Devolder, M. Bosch Princep and I. D. Fabian, Stochastic optimal control of annuity contracts, Insurance: Mathematics and Economics, 33(2003), pp. 227-238.

9. M. Di Giacinto, S. Federico and F. Gozzi, Pension funds with a minimum guarantee: a stochastic control approach, Finance and Stochastic, (2010).

10. J. Gao, Stochastic optimal control of DC pension funds, Insurance: Mathematics and Economics, 42(2008), pp. 1159-1164.

11. S. Haberman and E. Vigna, Optimal investment strategies and risk measures in defined contribution pension schemes, Insurance: Mathematics and Economics, 31(2002), pp. 35-69.

12. B. H $\phi \mathrm{jgaard}$ and $\mathrm{E}$. Vigna, Mean-variance portfolio selection and efficient frontier for defined contribution pension schemes, Technical report R-2007-13, Department of Mathematical Sciences, Aalborg University, 2007.

13. R. Josa-Fombellida and J. Rincón-Zapatero, Mean-variance portfolio and contribution selection in stochastic pension funding, European Journal of Operational Research, 187(2008), pp. 120-137.

14. R. Korn and M. Krekel, Optimal portfolios with fixed consumption or income streams. Working paper, University of Kaiserslautern, 2001.

15. $\mathrm{D} . \mathrm{Li}$ and $\mathrm{W} .-\mathrm{L}$. Ng, Optimal dynamic portfolio selection: multiperiod mean-variance formulation, Mathematical Finance, 10(2000), pp. 387-406.

16. C. I. Nkeki, 2011, On optimal portfolio management of the accumulation phase of a defined contributory pension scheme. Ph.D thesis, Department of Mathematics, University of Ibadan, Ibadan, Nigeria.

17. C. I. Nkeki and C. R. Nwozo, Variational form of classical portfolio strategy and expected wealth for a defined contributory pension scheme, Journal of Mathematical Finance 2(2012) 132-139.

18. C. I. Nkeki, Mean-variance portfolio selection problem with stochastic salary for a defined contribution pension scheme: A stochastic linear-quadratic framework, Statistics, optimization and information computing, 1(2013), 62-81.

19. S. Mannor and Tsitsiklis, J. N. Mean-variance optimization in Markov decision processes, Proceedings of the 28th international conference on machine learning Bellevue, WA, USA, (2011).

20. H. Richardson, A minimum variance result in continuous trading portfolio optimization, Management Science, 35(1989), pp. 1045-1055.

21. E. Vigna, On efficiency of mean-variance based portfolio selection in DC pension schemes, Collegio Carlo Alberto Notebook 154, 2010.

22. X. Zhou and $\mathrm{D}$. Li, Continuous-time mean-variance portfolio selection: A stochastic $L Q$ framework, Applied Mathematics and Optimization, 42(2000), pp. 19-33. 